\title{
Jamming Transition and Inherent Structures of Hard Spheres and Disks
}

\author{
Misaki Ozawa, Takeshi Kuroiwa, Atsushi Ikeda, ${ }^{*}$ and Kunimasa Miyazaki \\ Institute of Physics, University of Tsukuba, Tennodai 1-1-1, Tsukuba 305-8571, Japan
}

(Received 8 August 2012; published 13 November 2012)

\begin{abstract}
Recent studies show that volume fractions $\varphi_{J}$ at the jamming transition of frictionless hard spheres and disks are not uniquely determined but exist over a continuous range. Motivated by this observation, we numerically investigate the dependence of $\varphi_{J}$ on the initial configurations of the parent fluid equilibrated at a volume fraction $\varphi_{\mathrm{eq}}$, before compressing to generate a jammed packing. We find that $\varphi_{J}$ remains constant when $\varphi_{\mathrm{eq}}$ is small but sharply increases as $\varphi_{\mathrm{eq}}$ exceeds the dynamic transition point which the mode-coupling theory predicts. We carefully analyze configurational properties of both jammed packings and parent fluids and find that, while all jammed packings remain isostatic, the increase of $\varphi_{J}$ is accompanied with subtle but distinct changes of local orders, a static length scale, and an exponent of the finite-size scaling. These results are consistent with the scenario of the random first-order transition theory of the glass transition.
\end{abstract}

DOI: 10.1103/PhysRevLett.109.205701

PACS numbers: 64.70.P-, 45.70.Cc, 61.43.Fs

Despite their apparent similarities, a unifying theory of the glass transition of supercooled fluids and the jamming transition of athermal particles such as granular materials is still missing. Both are characterized by a transition from a flowing state to a randomly jammed state at a finite density or temperature. The glass transition is achieved by cooling equilibrium fluids slowly (but quickly enough to avoid crystallization), whereas a common protocol to induce the jamming transition is to compress dilute hardsphere or disk systems rapidly. For frictionless particle systems (which we shall consider in this Letter), it has long been argued that the jamming transition is interpreted as the zero-temperature limit of the glass transition [1]. Numerical studies, however, show that these two transitions are distinct and their natures are more complicated [2]. For example, the jamming transition has been believed to take place sharply at a unique volume fraction in the thermodynamic limit, the so-called "points J": $\varphi_{J} \approx 64 \%$ for three dimensions (3D) and $84 \%$ for two dimensions (2D) [3]. But recently it has been demonstrated that $\varphi_{J}$ is not unique but exists over a continuous range of volume fractions whose values vary depending on the protocols used to generate the jammed states $[4-6] ; \varphi_{J}$ becomes larger than $64 \%$ or $84 \%$ if one prepares moderately dense systems or thermally equilibrated systems at low temperatures and then rapidly compresses to generate the jammed states. Surprisingly, the jammed configurations at different $\varphi_{J}$ are found to remain isostatic, lack a partial crystalline order, and therefore are not mixtures of ordered and "maximally random jammed" states [6,7].

On the other hand, our understanding of the glass transition is no better than that of the jamming transition. Even a mean-field picture of the glass transition has not been established. A promising candidate is the so-called random first-order transition (RFOT) theory, originally inspired by the mean-field theory of spin glasses [8,9]. Crudely speaking, RFOT integrates the energy landscape picture, the concept of the ideal glass transition, and the modecoupling theory (MCT) [10-12]. Despite its theoretical coherence, this RFOT-MCT scenario still remains controversial, partly due to the lack of impeccable numerical and experimental evidence.

The goal of this Letter is to provide numerical evidence that the protocol dependence of $\varphi_{J}$ is a natural consequence of the RFOT-MCT scenario and thus the scenario can unify the glass and jamming transitions of frictionless particles. The idea that the energy landscape of glasses is intimately related to the jamming transition is not new [4,13-15]. But, to the best of our knowledge, quantitative characterizations of the transition points, particle configurations, and the associated length scales have not been done so far. First, we shall briefly recapitulate the essence of the RFOT-MCT scenario [9] and how it relates the jamming to the glass transition [13]. In the mean-field limit, RFOT predicts that, as a fluid is cooled down, it first undergoes the dynamical transition at a temperature $T_{\text {mct }}$ followed by the thermodynamic transition at a lower temperature $T_{K}$. Below $T_{\text {mct }}$, the multidimensional energy surface becomes suddenly rugged. The energies at local minima of the surface or the inherent structures (IS), $e_{\mathrm{IS}}$, which are almost constant at high temperatures, start decreasing at $T_{\text {mct }}$. Concomitantly, the saddles of the energy surface vanish and all stationary points become stable. The dynamics near $T_{\mathrm{mct}}$ is described by MCT. It predicts that dynamical quantities such as the relaxation time $\tau_{\alpha}$ diverge with a power law $\left|T-T_{\text {mct }}\right|^{-\gamma}$, where $\gamma$ is a parameter also calculated by MCT [12]. In finite dimensions, however, the dynamic transition is smeared out by activation hoppings between local minima separated by finite barriers and becomes merely a crossover. An important observation is that the geometrical properties of the energy landscape are not controlled by a single temperature $T_{\text {mct }}$ any more. 
Simulations have revealed that $e_{\mathrm{IS}}$ starts decreasing abruptly at a onset temperature $T_{o}$, whereas saddles survive well below $T_{o}$ until they vanish at $T_{\mathrm{th}}$, a so-called threshold temperature $[16,17]$. On the other hand, the relaxation time obtained by simulations is still well fitted by MCT's power law $\tau_{\alpha} \sim\left|T-T_{\mathrm{mct}}^{(\mathrm{fit})}\right|^{-\gamma}$, but $T_{\mathrm{mct}}^{(\mathrm{fit})}$ used for fitting was found to be considerably lower than $T_{\mathrm{mct}}^{\text {(theor) }}$, the value obtained theoretically by solving the MCT equation [12]. Surprisingly, $T_{\mathrm{mct}}^{\text {(fit) }}$ turned out to be very close to $T_{\mathrm{th}}$ [16], whereas $T_{\mathrm{mct}}^{\text {(theor) }}$ is close to $T_{o}$ [17,18]. Discrepancies between $T_{\mathrm{mct}}^{(\mathrm{fit})}\left(\approx T_{\mathrm{th}}\right)$ and $T_{\mathrm{mct}}^{\text {(theor) }}\left(\approx T_{o}\right)$, both of which should be identical in the mean-field limit, are due to the non-mean-field effect and can be explained by using kinetic arguments [19,20].

The above argument also applies to hard-sphere fluids. The temperature and energy $\left(T, e_{\mathrm{IS}}\right)$, relevant variables for continuous potential fluids, should be replaced by the (inverse) pressure $P^{-1}$ and volume for hard-core potential systems [21]. Instead of the volume, we shall adopt the density, or volume fraction $\varphi$. The inherent structures $\varphi_{\text {IS }}$ are obtained by compressing a parent fluid equilibrated at a finite $P$ by letting $P \rightarrow \infty$ (with an extra minimization using a conjugate gradient method), just as $T$ is quenched to zero to obtain $e_{\mathrm{IS}}$ for continuous potential fluids. This is nothing less than a process to generate jammed packings for frictionless hard spheres, and thus $\varphi_{\mathrm{IS}}$ should be equivalent with $\varphi_{J}$. Employing the RFOT scenario discussed above, we predict that $\varphi_{\text {IS }}$ or $\varphi_{J}$ is unchanged as long as $P$ of the parent fluid equilibrated at a volume fraction $\varphi_{\mathrm{eq}}$ is low but starts increasing as $P$ (or $\varphi_{\mathrm{eq}}$ ) exceeds $P_{\text {mct }}$ (or $\varphi_{\text {mct }}$ ). In other words, $\varphi_{J}$ is not a unique value but is a function of $P$ or $\varphi_{\text {eq }}$ and can exist over a continuous range [13-15]. The largest $\varphi_{J}^{(\max )}$ would correspond to the inherent structures of the fluid at the thermodynamic transition point, i.e., $\varphi_{\mathrm{eq}}=\varphi_{K}$. For finite dimensional systems, $\varphi_{\text {mct }}^{\text {(theor) }}$ obtained from MCT theoretically should be lower than $\varphi_{\text {mct }}^{\text {(fit) }}$ obtained by fitting the simulation data for the relaxation times. Furthermore, $\varphi_{J}$ should increase if we prepare a dense parent fluid such that $\varphi_{\mathrm{eq}}>\varphi_{\mathrm{mct}}^{(\text {theor) }}$ (rather than $\varphi_{\mathrm{mct}}^{(\mathrm{fit})}$ ). Another important prediction of RFOT is that, at the dynamic transition point, the system enters the coexisting region of numerous metastable phases, or mosaics. Thus, the static length scale associated with the mosaics, if any, should appear at $\varphi_{\text {mct }}^{\text {(theor) }}$. There have been several attempts to directly measure the static length in supercooled fluids, but most studies have focused on the configurations of parent fluids and at far lower temperatures or higher densities than the dynamic transition points [9].

In order to verify these predictions, we prepare thermally equilibrated hard spheres (3D) and disks (2D) at various initial fractions $\varphi_{\mathrm{eq}}$ and study their inherent structures $\varphi_{J}$. Both systems studied here are 50:50 binary mixtures with a size ratio of 1.4 with periodic boundary conditions $[3,4]$. The systems are equilibrated at $\varphi_{\text {eq }}$ by using Monte Carlo simulation and then compressed rapidly to generate jammed states. Following the procedure employed in Refs. [3,22], we switch the hard-core potential with the soft harmonic potential just before the compression, allowing particles to overlap. The system is then relaxed to the zero-energy state by using the conjugate gradient method. This compression and energyminimization cycle is iterated till the volume fraction is maximized without particle overlap. Note that the algorithm to generate the jammed states is essential. For example, the Lubachevsky-Stillinger algorithm is inappropriate, because the system keeps equilibrating during the slow compression and finds lower local minima of the landscape or higher $\varphi_{J}$ [23]. The system sizes are varied from $N=64$ to 2048. $\varphi_{J}$ in the large $N$ limit is evaluated by using the finite-size scaling $\left|\varphi_{J}(N)-\varphi_{J}\right| \sim N^{-1 / \nu d}$, where $d$ is the spatial dimension. In Fig. 1, the dependence of $\varphi_{J}$ on $\varphi_{\text {eq }}$ is shown. The exponents $\nu=0.72$ (3D) and 0.74 (2D) obtained for the smallest $\varphi_{\mathrm{eq}}$ are used for the rest of data. Actually, we found that $\nu$ varies noticeably depending on $\varphi_{\text {eq }}$ as we shall discuss below. However, the different $\nu$ 's do not affect appreciably the results of Fig. 1, other than more scattering of data points and larger error bars. At small $\varphi_{\text {eq }}$, the jamming transition points are identical with those already reported in the literature: $\varphi_{J} \approx$ 0.648 (3D) and 0.842 (2D) [3]. However, $\varphi_{J}$ abruptly starts increasing at large $\varphi_{\text {eq }}$. The onset fractions are found to be very close to $\varphi_{\mathrm{mct}}^{\text {(theor) }}$ independently evaluated by solving the MCT equations for binary mixtures using the static structure factor matrix $S(k)$ obtained by the Percus-Yevick theory as an input. Indicated by arrows in Fig. 1 are $\varphi_{\text {mct }}^{\text {(theor) }} \approx 0.516(3 \mathrm{D})$ and $0.685(2 \mathrm{D})$. We confirmed that these values do not vary more than $2 \%$ if simulated $S(k)$ is used. The onset points are obviously much lower than $\varphi_{\mathrm{mct}}^{(\mathrm{fit})} \approx 0.59$ (3D) and 0.79 (2D) obtained from fitting the relaxation data [24,25]. Results shown in Fig. 1 are consistent with those reported in Refs. [4,6]. We also found that, as $\varphi_{J}$ increases, the jammed configurations remain
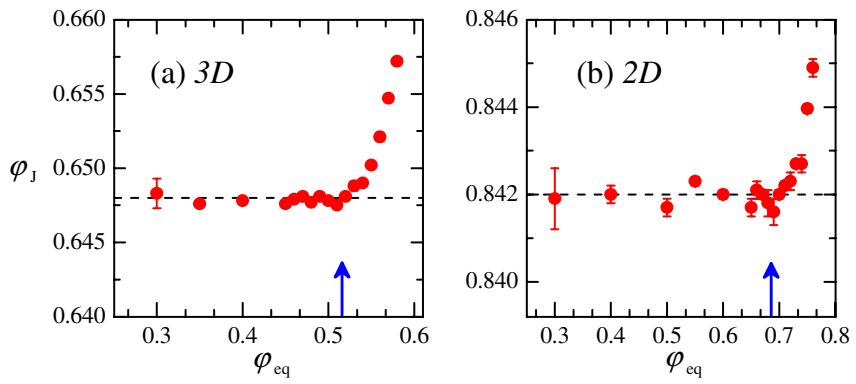

FIG. 1 (color online). $\varphi_{J}$ as a function of $\varphi_{\mathrm{eq}}$ for binary mixtures in (a) 3D and (b) 2D. Arrows indicate the positions of $\varphi_{\mathrm{mct}}^{\text {(ther) }}$. Broken horizontal lines are $\varphi_{J}$ reported by O'Hern et al. [3]. 
isostatic-i.e., the contact number at $\varphi_{J}$ is given by $z=2 d$-whereas the number of rattlers slightly increases [4]. We also measured the time sequence of the inherent structures for several $\varphi_{\mathrm{eq}}$ and observed that the patterns of sequences qualitatively change from white-noise-like at $\varphi_{\mathrm{eq}}<\varphi_{\mathrm{mct}}^{\text {(theor) }}$ to stepwise at $\varphi_{\mathrm{eq}}>\varphi_{\mathrm{mct}}^{\text {(theor) }}$ (not shown), implying that the nature of the landscape is altered [26]. These results support quantitatively that the jamming and glass transitions can be discussed under the common rubric of the RFOT-MCT scenario and also that $\varphi_{\text {mct }}^{\text {(theor) }}$ is not a fictitious value of an approximate theory but bears the essential geometrical meaning.

In order to clarify the nature of the denser jammed packings obtained from the parent fluid at $\varphi_{\mathrm{eq}}>\varphi_{\mathrm{mct}}^{\text {(theor) }}$, we focus on properties of their configurations. We calculate the compositional and orientational orders. Figure 2 shows the dependence on $\varphi_{\text {eq }}$ of the compositional order parameters of the jammed packings $\left(f_{L L}, f_{S S}\right.$, and $\left.f_{S L}\right)$, the number fractions of the contact pairs of the large $(L)$ and small $(S)$ particles [6]. For ideally random configurations, $f_{L L}+f_{S S} \approx f_{S L} \approx 0.5$ holds. Though this is the case for all $\varphi_{\text {eq }}$, a minute but sharp increase of $f_{L L}$ and a decrease of $f_{S S}$ are observed at $\varphi_{\mathrm{eq}} \approx \varphi_{\mathrm{mct}}^{\text {(theor) }}$. For 3D, the variations are about $5 \%$. Qualitatively similar changes are observed for 2D, consistent with Ref. [6]. We next analyze the bond-orientational order (BOO) parameters $Q_{4}$ and $Q_{6}$ (3D) and $\Psi_{6}$ (2D) defined in Refs. [6,27]. The BOO parameters evaluated for the large particles are shown in Figs. 3(a)-3(c). The results for the small particles show qualitatively similar behavior, although the variations are less pronounced. All results demonstrate that the BOO parameters are constant at $\varphi_{\mathrm{eq}}<\varphi_{\mathrm{mct}}^{\text {(theor) }}$ but change abruptly at $\varphi_{\mathrm{mct}}^{\text {(theor) }}$. One may want to argue that the synchronized change of $\varphi_{J}$ and the compositional or orientational orders is due to the onset of a partial crystallization or demixing and that the system traces a line connecting smoothly the maximally random jammed packing at the smallest $\varphi_{J}$ and the ideally ordered configuration at the maximal density [7]. If that is the case, however, the isostaticity should break down, and variations of $f_{i j}$ and the BOO parameters would be far larger than those shown in Figs. 2 and 3 [6]. Of course, we did not observe any sign of demixing from the eye inspection of the jammed configurations. These facts strongly suggest that the system is riding on a different branch. We emphasize that these sharp changes at $\varphi_{\text {mct }}^{\text {(theor) }}$ are observed only for the jammed packings. The inset in Fig. 3(c) shows that $\Psi_{6}$ of the parent fluid continuously increases with $\varphi_{\text {eq }}$ with no hint to change around $\varphi_{\mathrm{mct}}^{\text {(theor) }}$. Similar results were obtained for 3D.

According to RFOT, the increase of $\varphi_{J}$ should be accompanied by the appearance of numerous metastable states or mosaics and the system "phase separates" into these states. Thus, it is expected that the mosaics and their associated

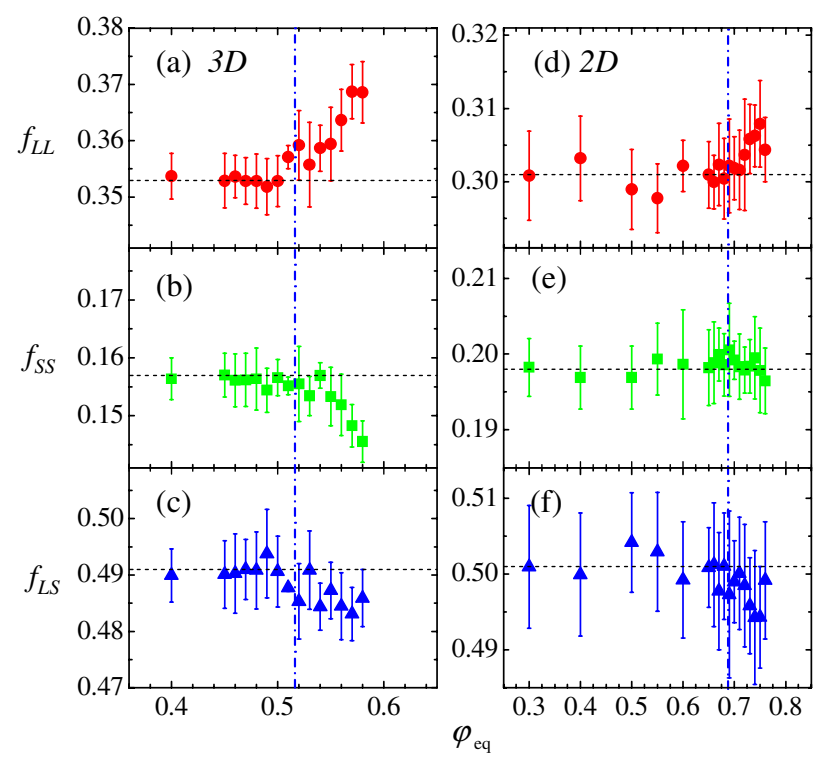

FIG. 2 (color online). Compositional order parameters for 3D (a)-(c) and 2D (d)-(f). Vertical dash-dotted lines represent $\varphi_{\text {mct }}^{\text {(theor) }}$, and horizontal broken lines are guides for the eye.

length scale should appear at $\varphi_{\text {mct }}^{\text {(theor) }}$. To detect a hint of the emergence of such states, we calculate the static correlation function of the fluctuations of the local $\mathrm{BOO}$ parameters $g_{6}(r)=\left\langle\delta \Psi_{6}(\mathbf{r}) \delta \Psi_{6}(0)\right\rangle$ for $2 \mathrm{D}$ and extract out the length scale $\xi_{6}$ by fitting the results with the Ornstein-Zernike function [Fig. 3(d)]. A similar result was obtained for 3D. $\xi_{6}$ which is constant at low $\varphi_{\mathrm{eq}}$ starts increasing at $\varphi_{\mathrm{mct}}^{\text {(theor) }}$. Also shown is $\xi_{6}$ obtained for the parent fluid, which monotonically increases with $\varphi_{\text {eq }}$. The sudden increase of $\xi_{6}$ at $\varphi_{\text {mct }}^{\text {(theor) }}$ for the jammed packing, which is not observed for the parent fluid, suggests a possibility that it is a direct reflection of the emergence of the mosaics.

Finally, we argue that $\varphi_{\mathrm{mct}}^{\text {(theor) }}$ may also mark the point beyond which the finite-size scaling law is qualitatively altered due to the emergence of mosaics. In the crossover region at which the MCT's critical dynamics and activation hoppings coexist, the finite-size effect is highly nontrivial according to the RFOT-MCT scenario [28]. For the shortrange interaction systems, these two mechanisms may compete and a simple power-law scaling may be violated. Figure 4 shows $\varphi_{\text {eq }}$ dependence of the finite-size scaling exponent $\nu$, obtained by naively using the scaling law. $\nu$ 's are constant at $\varphi_{\mathrm{eq}}<\varphi_{\mathrm{mct}}^{\text {(theor) }}$ and close to the values reported in Ref. [3] but start fluctuating and become errant at $\varphi_{\mathrm{eq}} \approx \varphi_{\mathrm{mct}}^{\text {(theor) }}$. We presume that this is another, though indirect, evidence supporting the RFOT-MCT scenario.

In summary, we have accumulated and displayed quantitative evidence that the RFOT-MCT scenario integrates the jamming and glass transitions in a common language and successfully explains the continuous increase of $\varphi_{J}$ reported previously. We demonstrated for the first time that 

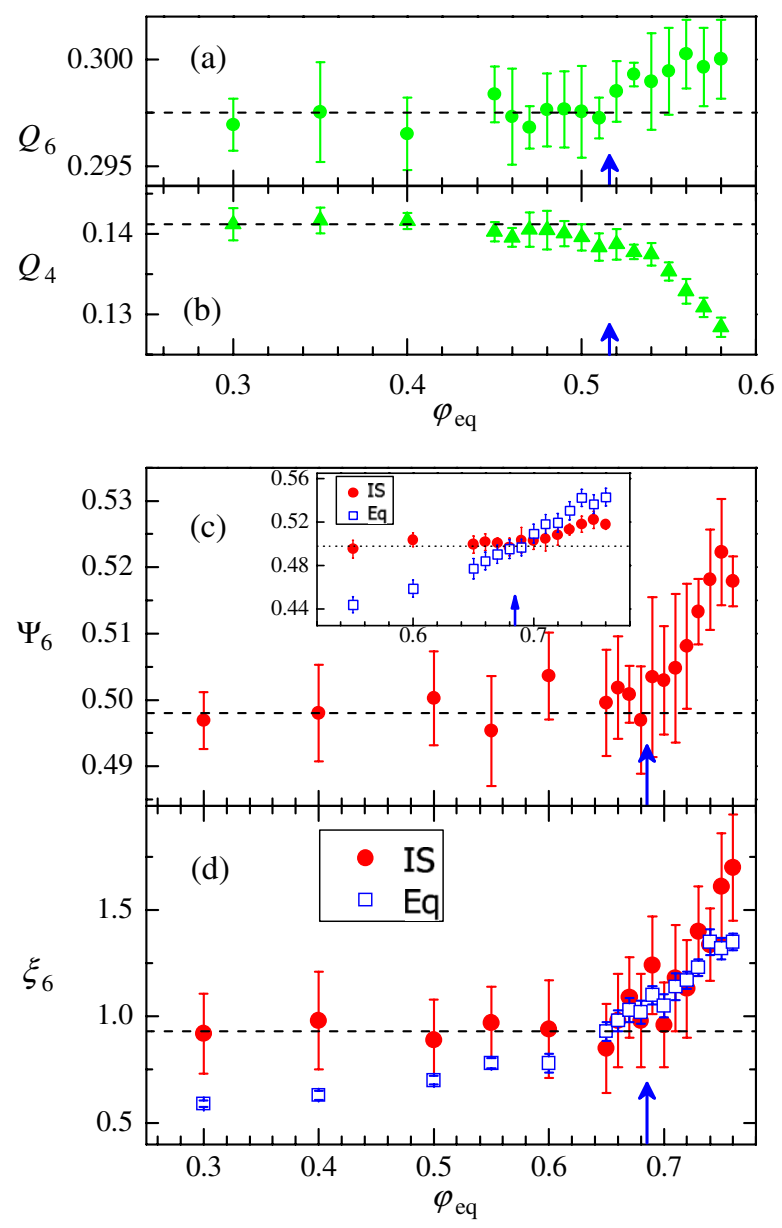

FIG. 3 (color online). BOO parameters for large particles of jammed packings (a) $Q_{6}$ and (b) $Q_{4}$ for $3 \mathrm{D}$ and (c) $\Psi_{6}$ for $2 \mathrm{D}$. The inset in (c) is $\Psi_{6}$ for the parent fluid (Eq; empty squares) and the jammed packing (IS; filled circles). (d) The correlation length $\xi_{6}$ obtained from $g_{6}(r)$ of the jammed packing and the parent fluid for 2D. Horizontal broken lines are guides for the eye.

the dynamical transition point $\varphi_{\mathrm{mct}}^{\text {(theor) }}$ theoretically evaluated, and not $\varphi_{\mathrm{mct}}^{\text {(fit) }}$ obtained by the fitting, unambiguously marks the onset of qualitative changes of the energy landscape or the "volume landscape" for hard spheres or disks. Note that the results shown here are consistent with those for various short-ranged potential systems [18] but not for the fully connected models [29]. In Ref. [29], the onset volume fraction at which $\varphi_{J}$ starts increasing is considerably smaller than $\varphi_{\text {mct }}^{\text {(theor) }}$ obtained from the simulated relaxation time in the mean-field regime. This contradictory result might be due to the long-ranged interaction of the model. Indeed, it is known that the onset temperature of the inherent structures for a fully connected spin-glass model of a finite size is much higher than the mean-field value and the convergence to the mean-field limit is extremely slow [30].

All results in this Letter eloquently support the RFOTMCT scenario, but many nagging questions are left for us.
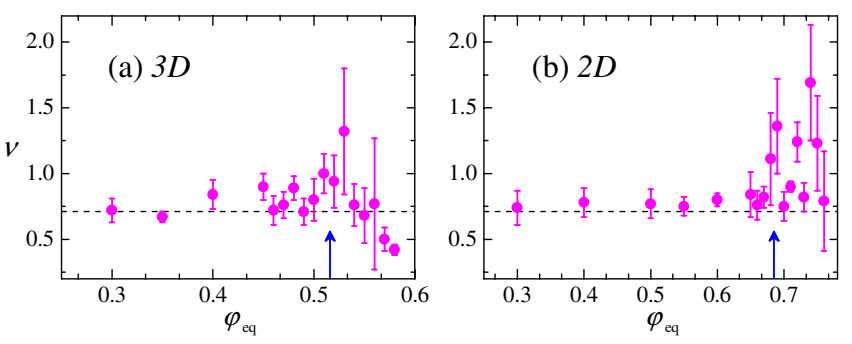

FIG. 4 (color online). $\varphi_{\text {eq }}$ dependence of $\nu$ of the finite-size scaling law $\left|\varphi_{J}(N)-\varphi_{J}\right| \sim N^{-1 / \nu d}$ for (a) 3D and (b) $2 \mathrm{D}$. The horizontal broken lines are values reported in Ref. [3].

For example, why does the MCT work quantitatively so well in finite dimensions? It is especially puzzling because recent studies show that the traditional MCT is not perfectly consistent with the mean-field scenario at large spatial dimensions [31]. Also, we are left unanswered about the relation of the static length which we observed with other lengths via static and dynamic measurements in the past $[9,32,33]$. And the last interesting question may be whether the configurational properties, especially the isostatic nature, of jammed packings are affected when $\varphi_{\text {eq }}$ exceeds $\varphi_{\text {mct }}^{\text {(fit) }}$ at which all saddles of the energy surface near the IS vanishes. These are a few of the many problems which are left for future work.

This work is supported by the JSPS Core-to-Core Program "International research network for nonequilibrium dynamics of soft matter," KAKENHI No. 2154016 and No. 24340098, and Priority Areas "Soft Matter Physics." We thank the Research Center for Computational Science, Okazaki and ISSP of Tokyo University for the use of supercomputers. We thank L. Berthier, P. Charbonneau, S. Sastry, and F. Zamponi for valuable discussions.

*Present address: Laboratoire Charles Coulomb, UMR 5221 CNRS, Montpellier, France.

[1] A. J. Liu and S. R. Nagel, Nature (London) 396, 21 (1998).

[2] A. Ikeda, L. Berthier, and P. Sollich, Phys. Rev. Lett. 109, 018301 (2012).

[3] C.S. O'Hern, L.E. Silbert, A. J. Liu, and S. R. Nagel, Phys. Rev. E 68, 011306 (2003).

[4] P. Chaudhuri, L. Berthier, and S. Sastry, Phys. Rev. Lett. 104, 165701 (2010).

[5] M. Pica Ciamarra, A. Coniglio, and A. de Candia, Soft Matter 6, 2975 (2010); M. Hermes and M. Dijkstra, Europhys. Lett. 89, 38005 (2010); D. Vågberg, P. Olsson, and S. Teitel, Phys. Rev. E 83, 031307 (2011).

[6] C. F. Schreck, C. S. O'Hern, and L. E. Silbert, Phys. Rev. E 84, 011305 (2011).

[7] S. Torquato, T. M. Truskett, and P. G. Debenedetti, Phys. Rev. Lett. 84, 2064 (2000).

[8] T. R. Kirkpatrick, D. Thirumalai, and P. G. Wolynes, Phys. Rev. A 40, 1045 (1989). 
[9] G. Biroli and J.P. Bouchaud, in Structural Glasses and Supercooled Liquids, edited by V. Lubchenko and P. Wolynes (Wiley, New York, 2012).

[10] M. Goldstein, J. Chem. Phys. 51, 3728 (1969).

[11] F. H. Stillinger and T. A. Weber, Phys. Rev. A 25, 978 (1982).

[12] W. Götze, Complex Dynamics of Glass-Forming Liquids (Oxford University, New York, 2009).

[13] R. Mari, F. Krzakala, and J. Kurchan, Phys. Rev. Lett. 103, 025701 (2009).

[14] G. Parisi and F. Zamponi, Rev. Mod. Phys. 82, 789 (2010).

[15] P. Charbonneau, A. Ikeda, G. Parisi, and F. Zamponi, Phys. Rev. Lett. 107, 185702 (2011).

[16] L. Angelani, R. Di Leonardo, G. Ruocco, A. Scala, and F. Sciortino, Phys. Rev. Lett. 85, 5356 (2000); K. Broderix, K. K. Bhattacharya, A. Cavagna, A. Zippelius, and I. Giardina, ibid. 85, 5360 (2000); T. S. Grigera, A. Cavagna, I. Giardina, and G. Parisi, ibid. 88, 055502 (2002).

[17] S. Sastry, P. G. Debenedetti, and F. H. Stillinger, Nature (London) 393, 554 (1998).

[18] Y. Brumer and D. R. Reichman, Phys. Rev. E 69, 041202 (2004).

[19] P. Mayer, K. Miyazaki, and D. R. Reichman, Phys. Rev. Lett. 97, 095702 (2006).

[20] S. M. Bhattacharyya, B. Bagchi, and P. G. Wolynes, Proc. Natl. Acad. Sci. U.S.A. 105, 16077 (2008).
[21] F. H. Stillinger, Jr., E. A. DiMarzio, and R. L. Kornegay, J. Chem. Phys. 40, 1564 (1964).

[22] K. W. Desmond and E. R. Weeks, Phys. Rev. E 80, 051305 (2009).

[23] B. D. Lubachevsky and F. H. Stillinger, J. Stat. Phys. 60, 561 (1990).

[24] G. Brambilla, D. El Masri, M. Pierno, L. Berthier, L. Cipelletti, G. Petekidis, and A. Schofield, Phys. Rev. Lett. 102, 085703 (2009).

[25] F. Weysser and D. Hajnal, Phys. Rev. E 83, 041503 (2011).

[26] R. A. Denny, D. R. Reichman, and J. P. Bouchaud, Phys. Rev. Lett. 90, 025503 (2003).

[27] P. J. Steinhardt, D. R. Nelson, and M. Ronchetti, Phys. Rev. B 28, 784 (1983).

[28] L. Berthier, G. Biroli, D. Coslovich, W. Kob, and C. Toninelli, Phys. Rev. E 86, 031502 (2012).

[29] R. Mari and J. Kurchan, J. Chem. Phys. 135, 124504 (2011).

[30] A. Crisanti and F. Ritort, Europhys. Lett. 51, 147 (2000).

[31] A. Ikeda and K. Miyazaki, Phys. Rev. Lett. 104, 255704 (2010).

[32] M. Mosayebi, E. Del Gado, P. Ilg, and H. C. Öttinger, Phys. Rev. Lett. 104, 205704 (2010); J. Chem. Phys. 137, 024504 (2012).

[33] H. Tanaka, J. Phys. Condens. Matter 23, 284115 (2011). 